antipsychotic medications are effective and recommended treatment for active psychotic symptoms, ${ }^{1}$ though there is not so much evidence for the long term (i.e. several years of antipsychotic treatments ${ }^{3}$ ). Additionally, the clinical use of these medications is not always straightforward because of their known side-effects and the fact that, in all psychiatric disorders and other illnesses in medicine, there are always patients who do not want to take the recommended treatment. This seems to have been the case in the trial pointed out by Bindman \& Kripalani. ${ }^{4}$ When considering the long-term effects of antipsychotics, it is evident that the long-term treatment of psychosis needs to be developed further.

We agree that it would be dangerous to see different treatments as alternatives to each other, and it has been shown that in psychiatry a combination of different treatments is, in general, more effective than any of them alone. ${ }^{5}$ Psychotherapy in the early phase of illness could be effective not only in preventing psychosis at prodromal phase, but also in enhancing adherence to antipsychotic medication. ${ }^{1}$ Current treatment guidelines do not suggest that treatment of first-episode psychosis should include only antipsychotic medication without psychosocial treatment, but rather state that medication is one of the cornerstones of psychosis treatment. We believe there is still a lot to do in developing both medication and psychosocial treatments for schizophrenia, and hopefully active research can support this development.

1 National Institute for Health and Care Excellence. Psychosis and Schizophrenia in Adults: Treatment and Management (Clinical Guideline 178). NICE, 2014.

2 de Haan L, Linszen DH, Lenior ME, de Win ED, Gorsira R. Duration of untreated psychosis and outcome of schizophrenia: delay in intensive psychosocial treatment versus delay in treatment with antipsychotic medication. Schizophr Bull 2003; 29: 341-8.

3 Harrow M, Jobe TH, Faull RN. Does treatment of schizophrenia with antipsychotic medications eliminate or reduce psychosis? A 20-year multi-follow-up study. Psychol Med 2014; 44: 3007-16.

4 Morrison AP, Turkington D, Pyle M, Spencer H, Brabban A, Dunn G, et al. Cognitive therapy for people with schizophrenia spectrum disorders not taking antipsychotic drugs: a single-blind randomised controlled trial. Lancet 2014; 383: 1395-403.

5 Cuijpers P, van Straten A, Warmerdam L, Andersson G. Psychotherapy versus the combination of psychotherapy and pharmacotherapy in the treatment of depression: a meta-analysis. Depress Anxiety 2009; 26: 279-88.

Matti Penttilä, Oulu University Institute of Clinical Medicine and Oulu University Hospital, email: Matti.Penttila@oulu.fi; Erika Jääskeläinen, Noora Hirvonen, Matti Isohanni, Oulu University Hospital, Jouko Miettunen, Oulu University Hospital and Medical Research Center Oulu, Finland.

doi: 10.1192/bjp.205.6.499a

\section{Electronic monitoring of forensic patients}

Tully et al raise important questions about the introduction of electronic monitoring of forensic patients. ${ }^{1}$ Incidents of absconding by forensic patients can give rise to calls for increased security and surveillance. As the authors point out, adoption of electronic monitoring as a panacea for these problems is shortsighted. Tully et al cover many of the concerns about electronic monitoring but one area is missing: that the evidence we have from electronic monitoring in the criminal justice sector is primarily of its effects on recidivism and absence without leave during use; evidence is very limited on the effects after its use.

In other words, electronic monitoring must eventually cease. Is the use of electronic monitoring during community reintegration actually preparing the patient for greater freedom and their rehabilitation, or simply delaying reoffending? Criminal justice experience with electronic monitoring focuses almost entirely on its effectiveness during use, such as on bail or as an alternative to incarceration, usually combined with home detention. Electronic monitoring combined with home detention is superior to imprisonment in these studies, but we already know that noncustodial responses to crime in general have superior outcomes to incarceration (see, for example, Wermink et $a l^{2}$ ).

We know very little about outcomes after the use of electronic monitoring. Although the use of global positioning satellite (GPS) technology might improve the person's performance in following rules, it is not clear that this sort of rule following encourages the person in the ultimate tasks of forensic rehabilitation. Does it improve the therapeutic alliance to help the person make the life changes necessary to recover from illness and illness-related offending? Or does electronic monitoring seem a physical manifestation of distrust and create distance between the patient and the treatment team? If the only way that a person can safely have community contact is to wear an ankle bracelet, isn't it questionable whether they are ready for that level of community contact? Electronic monitoring may allow the person more apparent personal freedom than their clinical risk would otherwise allow. As Tully et al point out, adoption of the GPS technology may seem appealing, but its costs and effects are not clear and neither is its impact on therapeutic and community engagement. Short-term reductions in absence without leave might give the appearance of progress that the patient has not actually achieved. Long-term outcome is equally as important as short-term adherence.

1 Tully J, Hearn D, Fahy T. Can electronic monitoring (GPS 'tracking') enhance risk management in psychiatry? Br J Psychiatry 2014; 205: 83-5.

2 Wermink H, Blokland A, Nieuwbeerta P, Nagin D, Tollenaar N. Comparing the effects of community service and short-term imprisonment on recidivism: a matched samples approach. J Exp Criminol 2010; 6: 325-49.

Alexander I. F. Simpson, Chief of Forensic Psychiatry, Stephanie R Penney, Centre for Addiction and Mental Health, email: sandy.simpson@camh.ca

doi: 10.1192/bjp.205.6.500

Tully and colleagues ${ }^{1}$ justify the introduction of electronic monitoring of medium secure patients without indication of the size of the problem of absconding or the incidence of serious harm other than to reference an article in The Sun newspaper, which is neither informative nor free of bias.

Decisions around leave for patients detained within a medium secure unit are clearly complex. Consideration should always be given to the risk of absconding and associated risks if the patient were to abscond. Thus, patients who are at high risk of absconding and a serious risk to the public would not receive leave, whether they were tagged or not. Another factor is the clinical team's trust in that patient to use leave appropriately. Tagging patients would be a very clear indicator of a lack of such trust.

The suggestion that patients enter into electronic monitoring with consent is questionable: many patients in our experience abide by suggestions of their clinical team in order to progress through the system. Given that there is yet to be a strong argument that tagging is necessary and primarily in the patient's best interest (as opposed to a matter of public protection), can one justify this coercion? We would be very interested to know the process in which patients' perspectives were taken into account and whether this has altered the intervention.

Electronic monitoring would inform the clinical team if the patient were to breach the conditions of their leave in terms of approximate location and time of leave; however, it would not inform the team as to what that patient was doing with their leave and would not necessarily prevent serious incidents occurring, as 